\title{
Combined aortic and inferior vena cava injury
}

\author{
Basem Marcos, Yomayra Perez, Jennifer Matarlo, Jay A Yelon, \\ Valerie Katz, Robert V Madlinger
}

\begin{abstract}
Introduction: Combined injuries to the aorta and inferior vena cava are among the most severe traumatic injuries where mortality rates can approach $100 \%$. Case Report: A 26-year-old male presented with multiple small caliber gunshot wounds to the right upper and lower back, right posterior arm and right gluteal area. He was diagnosed with large retroperitoneal hematoma on computed tomography (CT) scan. He underwent an exploratory laparotomy with exploration of a zone 1 retroperitoneal hematoma. An injury to the inferior vena cava (IVC) at the level of the left renal vein was identified and repaired by lateral venorrhaphy. Massive transfusion protocol (MTP) was activated. The patient was returned back to the operating room few hours later for recurrent bleeding. Aortic injury was identified one centimeter distal to the renal arteries secondary to ruptured pseudoaneurysm. The left renal vein
\end{abstract}

Basem Marcos ${ }^{1}$, Yomayra Perez², Jennifer Matarlo³, Jay A Yelon $^{4}$, Valerie Katz ${ }^{5}$, Robert V Madlinger ${ }^{6}$

Affiliations: ${ }^{1}$ Resident, Department of Surgery, Lincoln Medical and Mental Health, Bronx, New York, USA; ${ }^{2}$ Research Assistant, Department of Surgery, Lincoln Medical and Mental Health, Bronx, New York, USA; ${ }^{3}$ Trauma Coordinator, Department of Surgery, Lincoln Medical and Mental Health, Bronx, New York, USA; ${ }^{4}$ Chairman, Department of Surgery, Lincoln Medical and Mental Health, Bronx, New York, USA; ${ }^{5}$ Associate Program Director, Department of Surgery, Lincoln Medical and Mental Health, Bronx, New York, USA; ${ }^{6}$ Director of Trauma Services, Department of Traumatology, Lincoln Medical and Mental Health, Bronx, New York, USA.

Corresponding Author: Basem Marcos, 234 East 149th, Bronx, New York, USA. 10451; Ph: 718-579-5900; Fax: 718-579-4620; Email: basemsamir@yahoo.com

Received: 26 October 2011

Accepted: 24 April 2012

Published: 01 January 2013 was ligated for exposure. The aortic injury was repaired. The patient was resuscitated postoperatively and was discharged home without major morbidity. Conclusion: Combined inferior vena cava and infrarenal aortic injury carries a mortality of around $73 \%$. All zone 1 retroperitoneal hematomas should be explored, with the exception of venous hematomas of the juxtahepatic vena cava. Mattox maneuver is the ideal exposure for a supramesocolic retroperitoneal hematoma and Cattell-Brash maneuver is used for inframesocolic retroperitoneal hematomas. Zone 1 retroperitoneal hematoma resulting from combined injuries to the inferior vena cava and aorta is highly lethal. Successful management requires early MTP, rapid and aggressive surgical management and application of elective vascular surgery techniques.

Keywords: Inferior vena cava injury, Aorta injury

$* * * * * * * * *$

Marcos B, Perez Y, Matarlo J, Yelon JA, Katz V, Madlinger RV. Combined aortic and inferior vena cava injury. International Journal of Case Reports and Images 2013;4(1):51-54.

$$
* * * * * * * * *
$$

doi:10.5348/ijcri-2013-01-258-CR-12

\section{INTRODUCTION}

Combined injuries to the aorta and inferior vena cava (IVC) are among the most severe traumatic injuries and are associated with high mortality rates. Mortality rates for such injuries can approach 100\%. Adherence to 
advanced trauma life support protocol, principles of damage control surgery, and implementation of an evidence based massive transfusion protocol (MTP) may lead to improved survival with a decrease in major morbidity.

\section{CASE REPORT}

A 26-year-old male was brought to the emergency department (ED) by emergency medical services after he was found with multiple small caliber gunshot wounds to the right upper and lower back, right posterior arm and right gluteal area (Figure 1). On arrival his vital signs were: heart rate $72 / \mathrm{min}$, respiratory rate $22 / \mathrm{min}$, blood pressure $100 / 48 \mathrm{mmHg}$ and $\mathrm{spO} 2100 \%$. He complained of abdominal pain. On physical examination the abdomen was soft, nondistended and moderately tender over the midabdomen without rebound, rigidity, or guarding. Bowel sounds were present. Focused abdominal sonography for trauma (FAST) was performed in the ED which did not reveal any free fluid. Further diagnostic studies included a computed tomography (CT) scan of the abdomen and pelvis was suspicious for injuries to the abdominal aorta and portal vein.

The patient was immediately taken for operation. The MTP was activated in preparation for the possibility of a major abdominal vascular injury. Massive transfusion protocol in our institution is constituted of pRBCs:FFPs in 1:1 ratio followed by giving single donor platelets and 10 units of cryoprecipitate after 10 units each of pRBCs and FFPs. The patient underwent an exploratory laparotomy with exploration of a zone 1 retroperitoneal hematoma (Figure 2). An injury to the IVC at the level of the left renal vein was identified and repaired by lateral venorrhaphy. No bleeding was noted from the portal vein or the aorta. Hemorrhage was controlled, the abdomen was packed and left open because of mid-gut edema and the patient was transferred to the surgical intensive care unit (SICU) for continued resuscitation. A few hours later, the patient developed signs of hypovolemic shock and was immediately returned to the operating room. The MTP was again activated. Supraceliac aortic control was obtained and an aortic injury was identified one
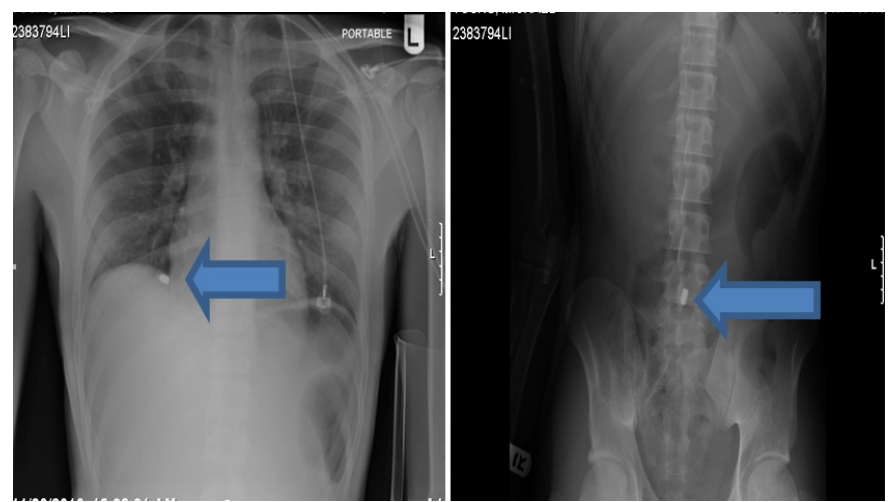

Figure 1: Plain chest and abdominal X-ray showing radioopaque bullet fragments.

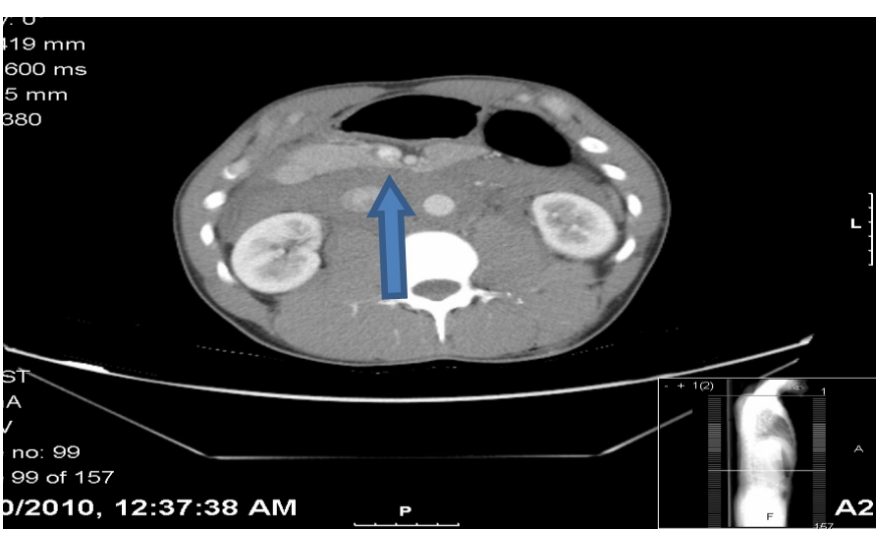

Figure 2: Computed tomography scan of the abdomen showing large zone 1 retroperitoneal hematoma.

centimeter distal to the renal arteries (this was the pseudoaneurysm that was present on the initial imaging and missed during the first laparotomy and progressed to subsequent rupture). The left renal vein was ligated and divided to provide adequate exposure of the injury. The aortic injury was repaired by primary suture closure. The patient received a total of 53 units of packed red blood cells, 49 units of fresh frozen plasma, 7 units of single donor platelets, and 40 units of cryoprecipitate perioperatively. At the end of surgery, the patient's lactate was $9.5 \mathrm{mmol} / \mathrm{L}$ and his base deficit was 18. Resuscitation was continued and these findings of hypoperfusion were corrected in less than 24 hours.

The patient underwent a planned re-exploration and abdominal washout with partial fascial closure on postoperative day (POD)-3. The abdomen was closed on POD-7. The patient developed acute cholecystitis and underwent an open cholecystectomy on POD-11. A tracheostomy was performed on POD-14. The patient spent 23 days in the SICU, four days in the surgical ward and he was then transferred to an acute inpatient rehabilitation center. The patient was seen in the surgery clinic following his discharge from inpatient rehabilitation. He was ambulating and tolerating a diet. His tracheostomy had been removed and the site of his tracheostomy.

\section{DISCUSSION}

The retroperitoneum is divided to zone 1 (around the abdominal aorta and IVC), zone 2 (around both the kidneys), and zone 3 (in the pelvis). Zone 1 retroperitoneal hematoma is a sign of a major abdominal vascular injury. Penetrating trauma accounts for approximately $90 \%$ cases. The overall mortality rate is $57 \%$. Mortality rates range from $30 \%$ for an infrarenal IVC injury, 50\% for an infrarenal aortic injury, $60 \%$ for an injury to the suprarenal abdominal aorta and $100 \%$ for combined injuries to the suprarenal abdominal aorta and IVC. Our patient had an injury to the IVC as well as an infrarenal aortic injury, which carries approximately $73 \%$ mortality rate. Factors associated with increased 
mortality are presence of shock at the time of admission, bleeding without retroperitoneal tamponade, acidosis, and suprarenal injury [1].

This injury is more common in urban trauma centers than it is in military conflicts. Debakey et al. reviewed 2,471 patients with arterial injuries during World War II. Two percent patients had abdominal arterial injuries. Rich et al. reviewed 1,000 patients with arterial injuries sustained in the Vietnam War. Only 2.9\% injuries involved abdominal vessels. A thirty-year review of 5,760 cardiovascular injures seen at Ben Taub Hospital (Houston, TX, USA) found that $33.8 \%$ patients had abdominal vascular injuries. This difference is thought to be due to the increased wounding power of military weapons, delayed transport and torso body armor [2].

Patients can present clinically with one of two pictures: (i) contained hematoma with transient hypotension responding to crystalloid boluses, or (ii) free intraperitoneal rupture with marked hypotension which does not respond to fluid boluses. Patients with contained hematoma, like our patient, can remain hemodynamically normal until the hematoma is opened in the operating room. In one review, patients with a contained hematoma had a mean base deficit of 7.2 , mean transfusion of 8.6 units of blood, and a survival rate of $96 \%$. Those with free intraperitoneal rupture had a mean base deficit of 14.7 , mean transfusion of 15.1 units and $43 \%$ survival [2]. In patients with penetrating abdominal trauma to the back and flank who are hemodinamically normal, CT scan of the abdomen can be obtained for further evaluation of injuries prior to surgery [3].

In cases with penetrating abdominal trauma, standard trauma principles apply. These include exploration via a midline incision, four quadrant packing and evacuation of blood and blood clots. Proximal and distal vascular control is essential and can be achieved by a variety of vascular techniques, including direct digital pressure, pressure with laparotomy pads (packing) or pressure with sponge sticks. Enteric injuries are addressed with clamps or staples. An associated ureteral injury may be treated initially by ligating the ureter during damage control surgery.

All zone 1 retroperitoneal hematomas should be explored, with the exception of venous hematomas of the juxtahepatic vena cava [4]. A left medial visceral rotation (Mattox maneuver) is the ideal exposure for a supramesocolic retroperitoneal hematoma and the right medial visceral rotation or Cattell-Brash maneuver with kocherization of the duodenum is used for inframesocolic retroperitoneal hematomas [5].

After hemorrhage control of the injured aorta or IVC is obtained, exploration of the hematoma is begun at the highest point. Lateral repair (simple primary suture repair) of the aorta and IVC should be attempted. Complex vascular repairs can be performed if there is loss of part of the arterial wall, but this is time consuming. The left renal vein can be ligated and divided. This aids in exposure of injuries at this level. If IVC repair is not possible, the IVC can be ligated. Lower extremity fasciotomies may be needed along with aggressive resuscitation. The infrarenal aorta can be ligated if it cannot be repaired with axillary bifemoral extra-anatomic bypass performed later, if the patient survives [4].

\section{CONCLUSION}

Zone 1 retroperitoneal hematoma resulting from combined injuries to the inferior vena cava and aorta is highly lethal. Management should be individualized based on the patient's presentation. Key maneuvers are hemorrhage control and exposure. Successful management of acute traumatic injury to both the abdominal aorta and inferior vena cava requires early activation of massive transfusion protocol, rapid and aggressive surgical management, including damage control techniques and application of techniques and principles developed for elective vascular surgery.

$* * * * * * * * *$

\section{Author Contributions}

Basem Marcos - Substantial contributions to conception and design, Acquisition of data, Analysis and interpretation of data, Drafting the article, Revising it critically for important intellectual content, Final approval of the version to be published

Yomayra Perez - Analysis and interpretation of data, Revising it critically for important intellectual content, Final approval of the version to be published

Jennifer Matarlo - Analysis and interpretation of data, Revising it critically for important intellectual content, Final approval of the version to be published

Jay A Yelon - Analysis and interpretation of data, Revising it critically for important intellectual content, Final approval of the version to be published Valerie Katz - Analysis and interpretation of data, Revising it critically for important intellectual content, Final approval of the version to be published

Robert V Madlinger - Analysis and interpretation of data, Revising it critically for important intellectual content, Final approval of the version to be published

\section{Guarantor}

The corresponding author is the guarantor of submission.

\section{Conflict of Interest}

Authors declare no conflict of interest.

\section{Copyright}

(C) Basem Marcos et al. 2013; This article is distributed under the terms of Creative Commons Attribution 3.0 License which permits unrestricted use, distribution and reproduction in any means provided the original authors and original publisher are properly credited. (Please see www.ijcasereportsandimages.com /copyright-policy.php for more information.) 


\section{REFERENCES}

1. Coimbra R, Hoyt D, Winchell R, Simons R, Fortlage D, Garcia J. The Ongoing Challenge of Retroperitoneal Vascular Injuries. American Journal of surgery 1996 Nov;172(5):541-4.

2. Juan A Asencio, Donald D Trunkey. Current Therapy of Trauma \& Surgical Critical Care 2008:410.
3. F. Charles Brunicardi, Dana K Andersen, Timothy R Billiar, David L Dunn, John G Hunter. Schwartz Principles of Surgery 2010(7):fig7-2.

4. Hirshberg Asher, Kenneth Mattox. Top knife art \& craft of trauma surgery 2005;9:131-46.

5. Lenworth Jacobs, Stephen Luk. Advanced Trauma Operative Management. Surgical Strategies for Penetrating Trauma 2010: 257-72.
Access full text article on other devices

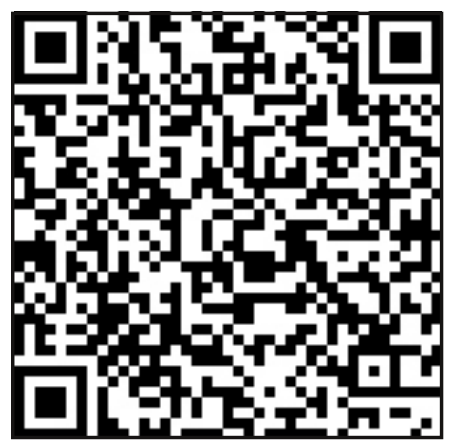

Access PDF of article on other devices

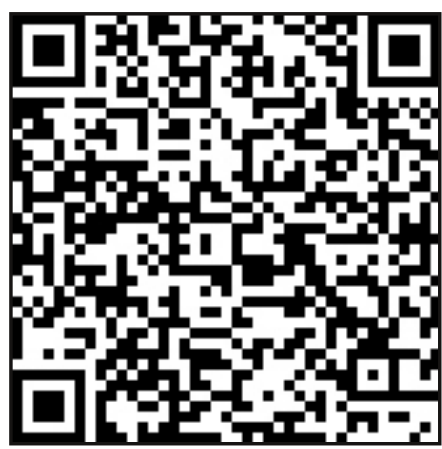

